\title{
11. PALEOMAGNETISM OF LEG 17 SEDIMENT CORES
}

\author{
Richard D. Jarrard, Scripps Institution of Oceanography, La Jolla, California
}

\section{METHODS}

Shipboard paleomagnetic sampling of freshly split cores was carried out on most undisturbed sediment cores recovered on Leg 17. Remanant magnetism of the 6-cc samples was measured either at $15 \mathrm{~Hz}$ on the PAR Spinner Magnetometer at Scripps or at $5 \mathrm{~Hz}$ on the spinner magnetometer at Stanford. Seven representative pilot samples were measured after alternating-field demagnetizations in fields of from 25 oe up to 200 or 300 oe (Table 1). Examination of variation of sample intensity, when plotted against the strength of the demagnetizing field (Figure 1), revealed that 50 oe is probably a suitable demagnetizing field for removal of most or all viscous magnetization. All samples were therefore measured before and after alternatingfield demagnetization in a peak field of 50 oe (Table 2).

Because of a bad amplifier, the Scripps spinner magnetometer has a very high noise level. Consequently many of the samples (indicated by asterisks in Table 2) were run on the Stanford spinner. Sample interval in Table 2 is the distance in centimeters from the top of the $2-\mathrm{cm}$ wide sample to the top of the section. Inclinations (Incl.) are given with respect to the present horizontal, positive downward. Declinations (Decl.) are relative only to a given core barrel; in many cases drilling has caused rotation of sediments within a core barrel by an unknown amount. MM and A are measures of the consistency of replicate measurements of intensity and direction of magnetization, respectively, on a single sample. Values of MM greater than about $20 \%$ or $30 \%$ or of A greater than about $5^{\circ}$ indicate that the measurements are probably unreliable, because of either very low intensity or instability of remanant magnetization. The last column in Table 2 records the appearance of bedding near the sampled interval and gives an indication of the reliability of the assumption of horizontal bedding in the sample. A indicates horizontal bedding directly above and below or within the sample. B indicates horizontal bedding directly above or below the sample. C indicates horizontal bedding somewhere in the section. D indicates slightly arched bedding directly above or below the sample. E indicates faint or no evidence of bedding.

Although some diabase and much basalt were recovered on Leg 17, no preliminary paleomagnetic measurements were carried out on igneous rocks. It is hoped that other investigations will do so.

\section{DISCUSSION}

Paleomagnetic measurements on basalts recovered by deep drilling on Midway Atoll indicate $13^{\circ} \pm 4^{\circ}$ of northward movement of the Pacific plate in the last $18 \mathrm{~m}$.y.
(Grommé and Vine, in press). Paleomagnetic evidence of Pacific plate motion since the Cretaceous is provided by topographic and magnetic surveys of seamounts (Francheteau et al., 1970); comparison of magnetic anomaly amplitudes on the two limbs of the Great Magnetic Bight (Vine, 1968); and comparison of magnetic anomalies in the Japanese, Hawaiian, and Phoenix anomaly groups (Larson and Chase, 1972). These three methods indicate about $30^{\circ}$ of northward motion of the Pacific plate in the past 70 to 110 m.y. Inclination of remanent magnetization in deep-sea drilling cores provides another measure of plate motion (Sclater and Cox, 1970); inclinations at Deep Sea Drilling Site 66 indicate about $30^{\circ}$ of northward motion of the Pacific plate since the Cretaceous (Sclater and Jarrard, 1971), consistent with other paleomagnetic evidence.

Figure 2 shows histograms of the inclinations (after 50 oe "cleaning") of samples from Table 2; samples of about the same age from a single site are grouped. Arrows in Figure 2 indicate the average inclinations expected if the site has moved northward $15^{\circ}$ or $30^{\circ}$. The Cretaceous samples from Site 167 show a strong grouping at about $10^{\circ}$ to $30^{\circ}$ inclination. Thus, the Site 167 data seem to indicate considerably less northward motion than do other Cretaceous paleomagnetic data for the Pacific plate. Anisotropic susceptibility studies are underway to determine whether this discrepancy could be accounted for by a gravityinduced inclination error in the Site 167 sediments.

Viscous magnetization may account for some of this discrepancy. Since these sediments were deposited south of the equator, viscous magnetization in the present earth's field would cause positive inclinations to increase and negative inclinations to decrease. Figure 2 shows that the positive inclinations tend to be steeper than the negative inclinations at Site 167. However, even the positive inclinations are lower than the $40^{\circ}$ inclination expected if $30^{\circ}$ of northward movement has occurred. Viscous magnetization would also cause higher average intensities for samples with negative inclinations than for those with positive inclinations. Such may be the case for the Site 167 Cretaceous sediments (Figure 3), but the marked increase in intensity with depth would tend to obscure this effect.

Figure 4 shows inclinations in Core 58 at Site 167. Two previously unreported magnetic reversals in the Coniacian are indicated by these samples. The Coniacian had previously been considered to be part of the Upper Cretaceous long period of normal polarity (Helsley and Steiner, 1969). McKenzie and Sclater (1971) indicate the presence of two reversed events near the beginning of anomaly 33 from sea-floor spreading evidence. If the Coniacian reversals correlate with the anomaly 33 events, then anomaly 33 is dated as about $85 \mathrm{~m} . \mathrm{y}$. 
TABLE 1

Demagnetization of Pilot Samples

\begin{tabular}{|c|c|c|c|c|c|c|c|}
\hline Sample & $\begin{array}{l}\text { Sample } \\
\text { Interval } \\
(\mathrm{cm})\end{array}$ & $\begin{array}{l}\text { Demag. } \\
\text { Field }\end{array}$ & $\begin{array}{c}\text { Incl. } \\
\text { (degrees) }\end{array}$ & $\begin{array}{c}\text { Decl. } \\
\text { (degrees) }\end{array}$ & $\underset{\text { (degrees) }}{\mathrm{A}}$ & $\begin{array}{c}\text { Intensity } \\
\times 10-5 \frac{\mathrm{EMU}}{\mathrm{cC}}\end{array}$ & $\begin{array}{l}\text { MM } \\
(\%)\end{array}$ \\
\hline $166-20-6$ & 88 & $\begin{array}{r}\text { NRM } \\
25 \\
50 \\
75 \\
100 \\
150\end{array}$ & $\begin{array}{l}-52.9 \\
-65.8 \\
-76.6 \\
-82.7 \\
-88.7 \\
-83.2\end{array}$ & $\begin{array}{r}325.4 \\
301.8 \\
297.1 \\
283.3 \\
251.9 \\
78.3\end{array}$ & $\begin{array}{l}3.3 \\
0.1 \\
0.2 \\
0.3 \\
0.0 \\
0.0\end{array}$ & $\begin{array}{l}16.2 \\
34.9 \\
37.7 \\
35.9 \\
35.5 \\
28.0\end{array}$ & $\begin{array}{r}12 \\
2 \\
2 \\
1 \\
2 \\
2\end{array}$ \\
\hline $166-20-1$ & 96 & $\begin{array}{r}\text { NRM } \\
25 \\
50 \\
75 \\
100 \\
150 \\
200\end{array}$ & $\begin{array}{r}-7.3 \\
-23.7 \\
-33.6 \\
-42.3 \\
-36.6 \\
29.3 \\
74.8\end{array}$ & $\begin{array}{l}267.0 \\
256.8 \\
252.5 \\
250.1 \\
235.1 \\
250.0 \\
239.9\end{array}$ & $\begin{array}{l}0.2 \\
0.0 \\
0.6 \\
0.2 \\
0.6 \\
1.3 \\
1.0\end{array}$ & $\begin{array}{r}39.4 \\
24.3 \\
15.3 \\
13.2 \\
10.4 \\
6.3 \\
6.4\end{array}$ & $\begin{array}{l}1 \\
3 \\
3 \\
3 \\
4 \\
6 \\
4\end{array}$ \\
\hline $164-10-4$ & 31 & $\begin{array}{r}\text { NRM } \\
25 \\
50 \\
75 \\
100 \\
150 \\
200 \\
250 \\
300\end{array}$ & $\begin{array}{r}71.7 \\
59.1 \\
46.7 \\
-46.2 \\
49.5 \\
61.3 \\
63.3 \\
72.7 \\
9.1\end{array}$ & $\begin{array}{l}133.1 \\
168.8 \\
170.4 \\
172.3 \\
166.7 \\
189.7 \\
153.2 \\
136.7 \\
198.6\end{array}$ & $\begin{array}{l}0.8 \\
0.1 \\
0.0 \\
0.4 \\
0.2 \\
0.2 \\
0.2 \\
0.0 \\
0.3\end{array}$ & $\begin{array}{l}32.0 \\
25.5 \\
25.1 \\
22.9 \\
19.3 \\
11.7 \\
23.2 \\
25.1 \\
43.9\end{array}$ & $\begin{array}{l}4 \\
1 \\
1 \\
3 \\
3 \\
4 \\
2 \\
1 \\
1\end{array}$ \\
\hline $166-27-2$ & 126 & $\begin{array}{r}\text { NRM } \\
25 \\
50 \\
75 \\
100 \\
150 \\
200\end{array}$ & $\begin{array}{r}-10.6 \\
-22.0 \\
-30.8 \\
-34.4 \\
-37.0 \\
-29.7 \\
2.8\end{array}$ & $\begin{array}{l}135.6 \\
153.9 \\
153.0 \\
160.4 \\
157.3 \\
160.3 \\
176.3\end{array}$ & $\begin{array}{l}0.0 \\
0.9 \\
0.4 \\
1.1 \\
0.4 \\
2.2 \\
0.8\end{array}$ & $\begin{array}{r}26.0 \\
11.4 \\
9.1 \\
6.8 \\
5.5 \\
4.2 \\
1.9\end{array}$ & $\begin{array}{r}5 \\
4 \\
7 \\
11 \\
10 \\
20 \\
21\end{array}$ \\
\hline $169-7-1$ & 70 & $\begin{array}{r}\text { NRM } \\
25 \\
50 \\
75 \\
100 \\
150 \\
200\end{array}$ & $\begin{array}{l}-17.1 \\
-24.1 \\
-29.1 \\
-29.9 \\
-31.8 \\
-37.8 \\
-18.2\end{array}$ & $\begin{array}{l}52.6 \\
56.5 \\
56.8 \\
56.7 \\
59.0 \\
56.8 \\
54.2\end{array}$ & $\begin{array}{l}0.0 \\
0.3 \\
0.3 \\
0.7 \\
0.5 \\
0.3 \\
0.1\end{array}$ & $\begin{array}{l}96.8 \\
87.3 \\
71.6 \\
60.0 \\
50.5 \\
37.8 \\
22.0\end{array}$ & $\begin{array}{l}1 \\
1 \\
1 \\
3 \\
2 \\
1 \\
2\end{array}$ \\
\hline $166-10-5$ & 140 & $\begin{array}{r}\text { NRM } \\
25 \\
50 \\
75 \\
100 \\
150 \\
200\end{array}$ & $\begin{array}{r}24.0 \\
17.2 \\
13.6 \\
10.9 \\
10.7 \\
9.0 \\
4.3\end{array}$ & $\begin{array}{l}201.2 \\
202.0 \\
199.1 \\
194.6 \\
197.2 \\
202.4 \\
202.9\end{array}$ & $\begin{array}{l}0.4 \\
0.1 \\
0.7 \\
0.9 \\
1.0 \\
0.3 \\
1.0\end{array}$ & $\begin{array}{l}7.5 \\
5.2 \\
4.8 \\
4.3 \\
3.5 \\
2.8 \\
2.1\end{array}$ & $\begin{array}{r}7 \\
5 \\
7 \\
9 \\
11 \\
9 \\
8\end{array}$ \\
\hline $167-57-1$ & 54 & $\begin{array}{r}\text { NRM } \\
25 \\
50 \\
75 \\
100 \\
150 \\
200\end{array}$ & $\begin{array}{r}-7.5 \\
-6.2 \\
-8.2 \\
-10.0 \\
-10.8 \\
-15.3 \\
-19.9\end{array}$ & $\begin{array}{l}339.1 \\
343.9 \\
344.3 \\
341.5 \\
343.5 \\
342.3 \\
339.0\end{array}$ & $\begin{array}{l}0.5 \\
0.0 \\
0.5 \\
0.5 \\
0.5 \\
0.4 \\
0.0\end{array}$ & $\begin{array}{l}8.0 \\
8.1 \\
7.6 \\
7.8 \\
7.2 \\
6.0 \\
5.2\end{array}$ & $\begin{array}{r}10 \\
9 \\
11 \\
10 \\
11 \\
11 \\
14\end{array}$ \\
\hline
\end{tabular}

NOTE: See text for definitions of A and MM. 

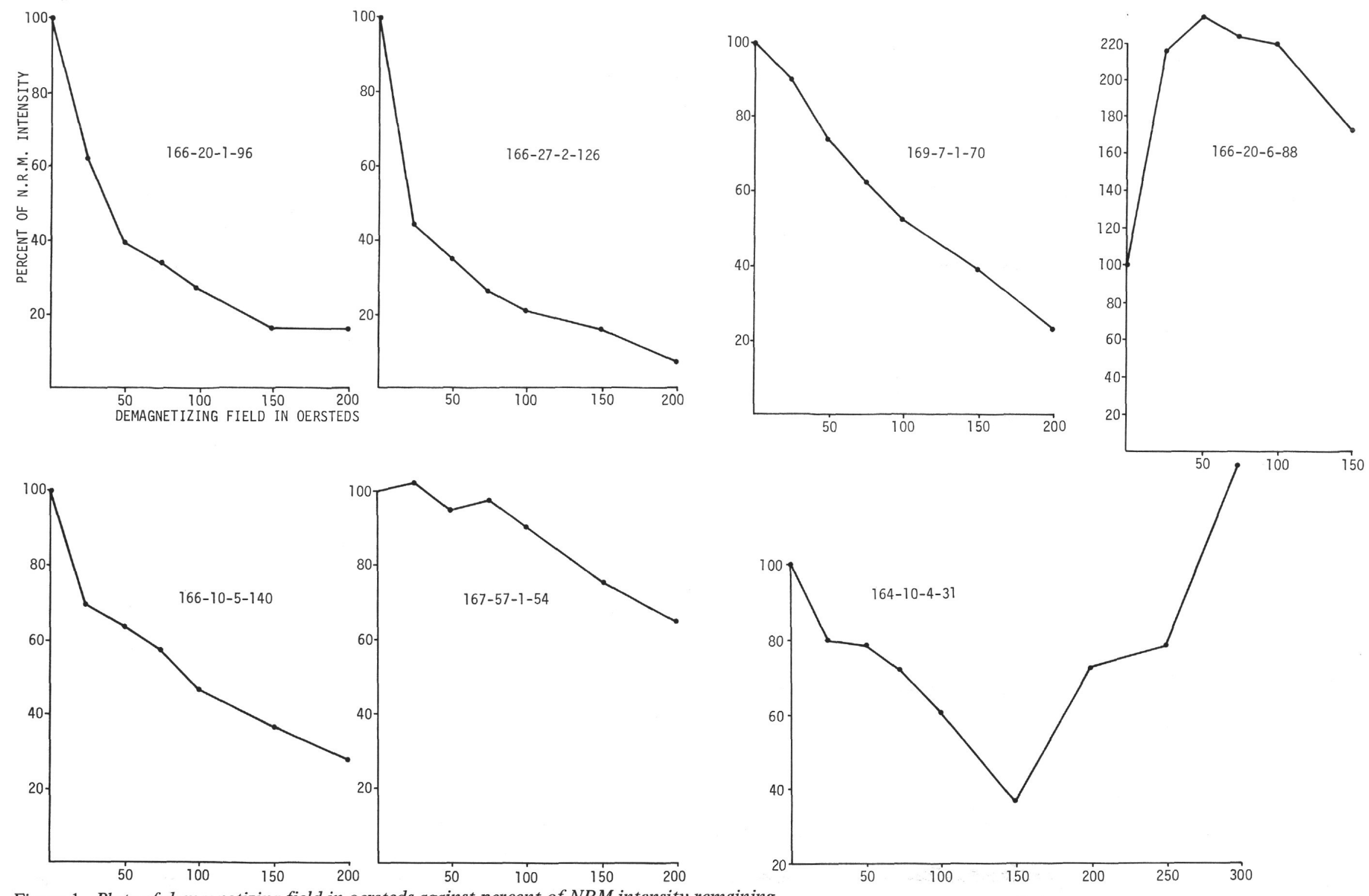

Figure 1. Plots of demagnetizing field in oersteds against percent of NRM intensity remaining. 
TABLE 2

Results of Paleomagnetic Measurements on Leg 17 Cores

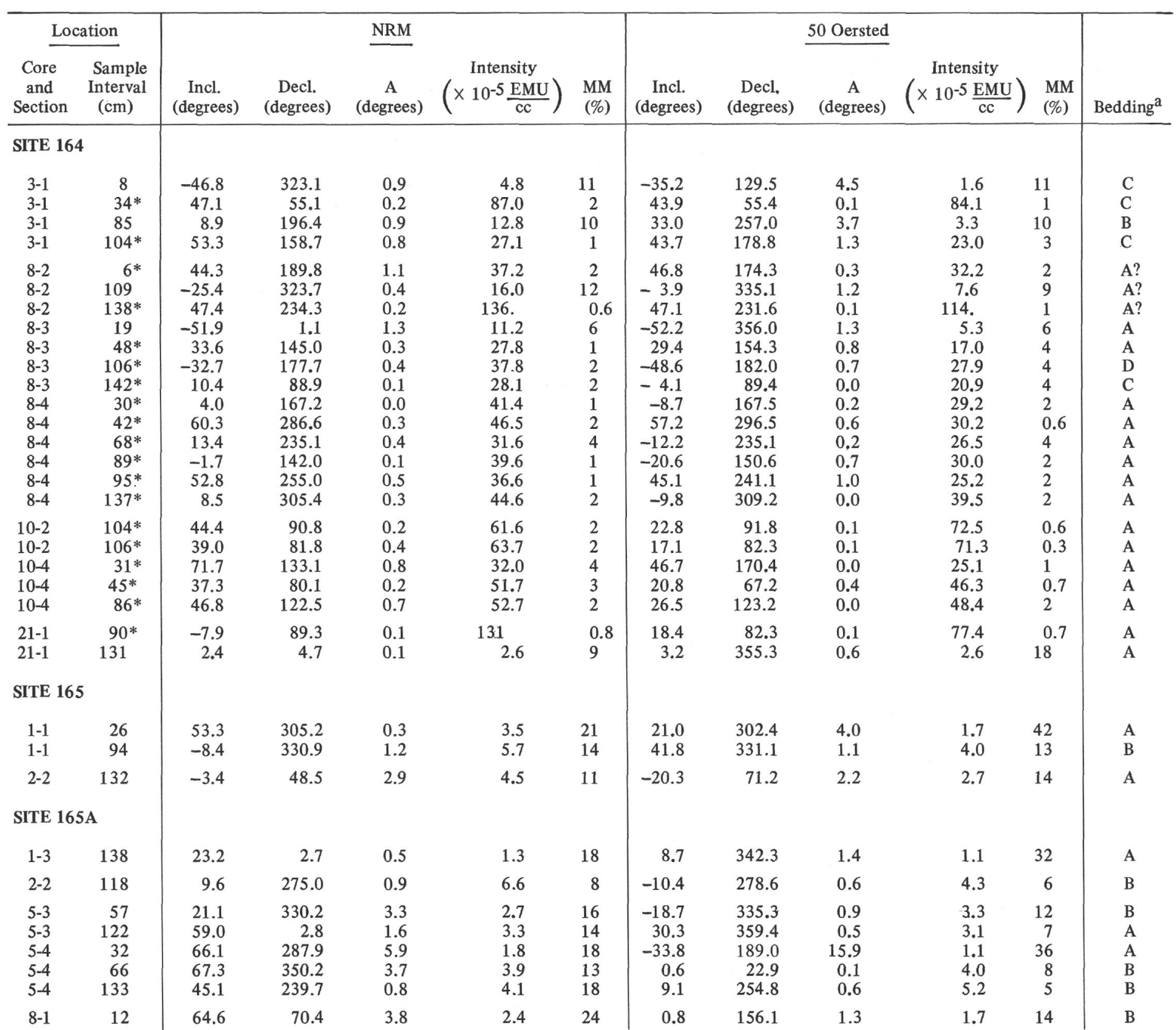




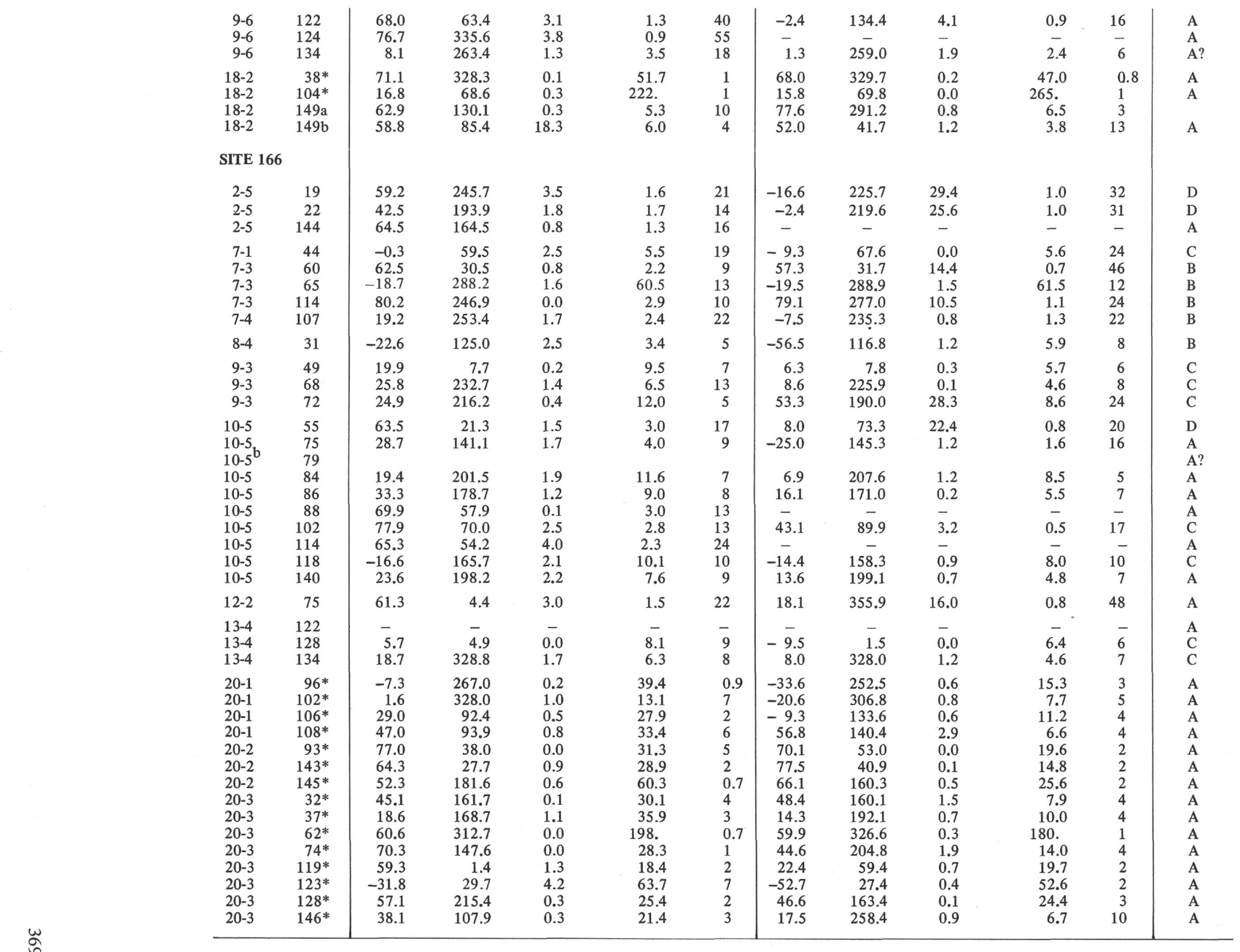




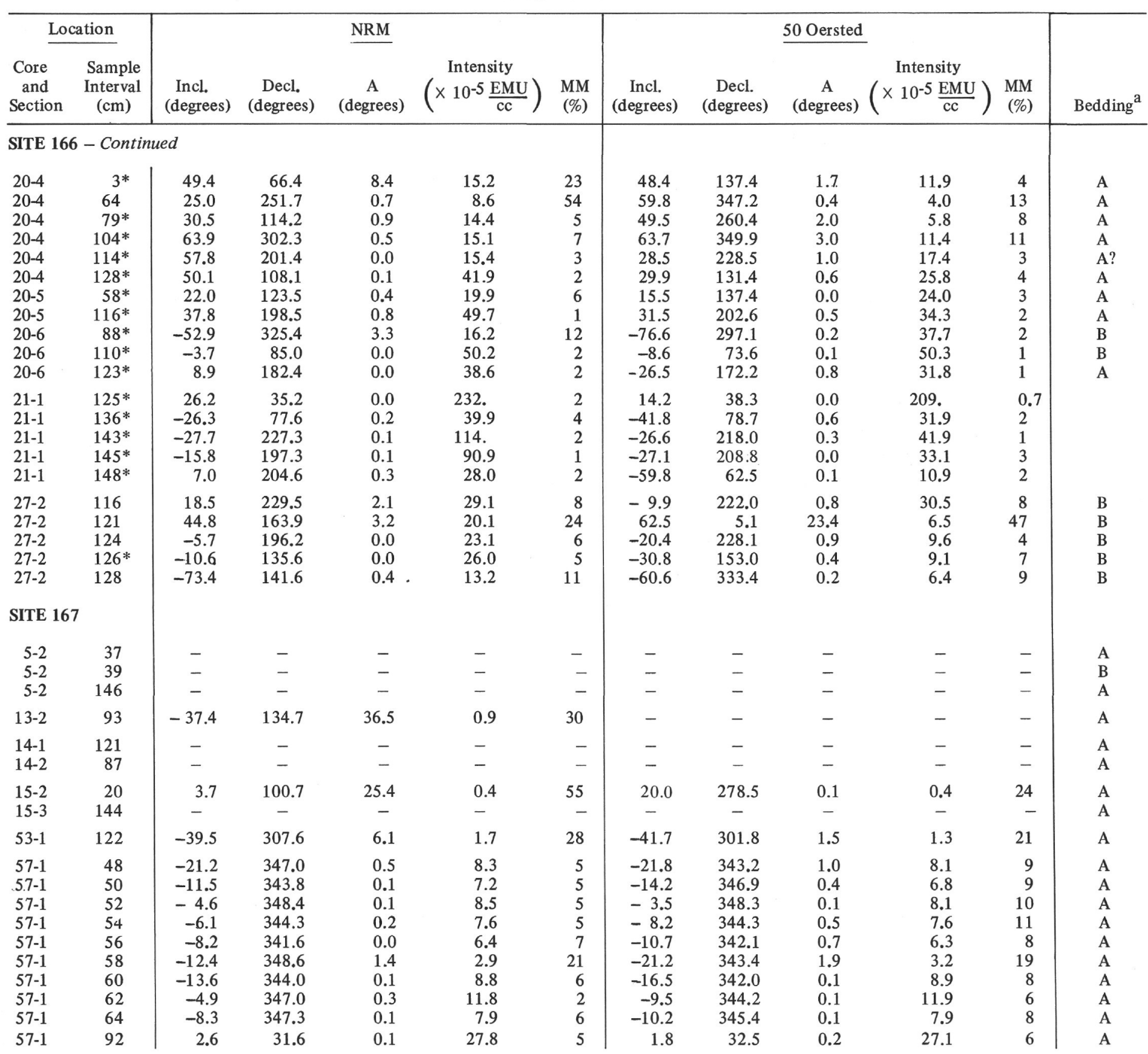




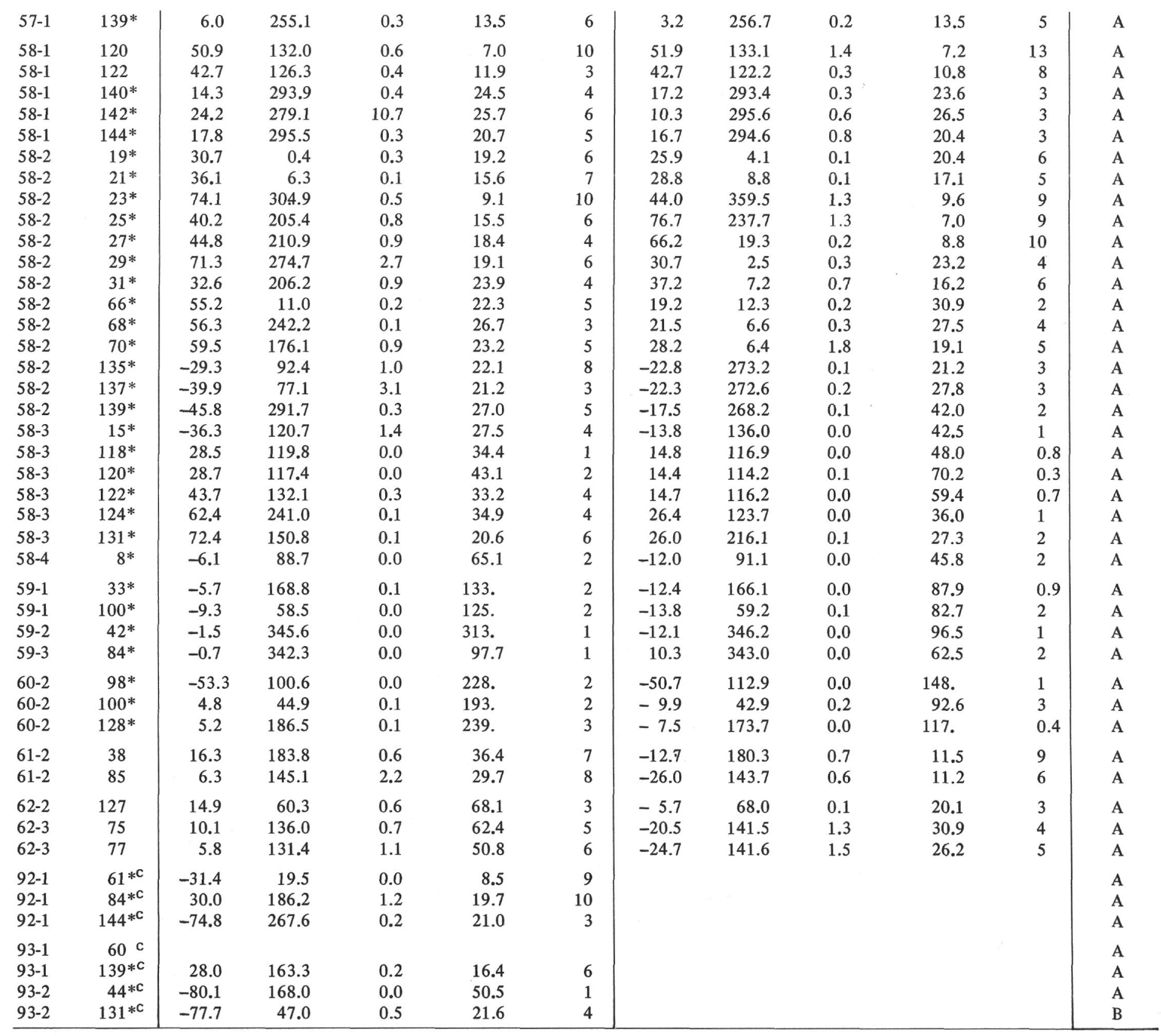


TABLE 2 - Continued

\begin{tabular}{|c|c|c|c|c|c|c|c|c|c|c|c|c|}
\hline \multicolumn{2}{|c|}{ Location } & \multicolumn{5}{|c|}{$\underline{N R M}$} & \multicolumn{5}{|c|}{$\underline{50 \text { Oersted }}$} & \multirow[b]{2}{*}{ Beddinga } \\
\hline $\begin{array}{l}\text { Core } \\
\text { and } \\
\text { Section }\end{array}$ & $\begin{array}{l}\text { Sample } \\
\text { Interval } \\
\text { (cm) }\end{array}$ & $\begin{array}{c}\text { Incl. } \\
\text { (degrees) }\end{array}$ & $\begin{array}{c}\text { Decl. } \\
\text { (degrees) }\end{array}$ & $\underset{\text { (degrees) }}{\mathrm{A}}$ & 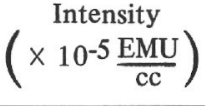 & $\begin{array}{l}\text { MM } \\
(\%)\end{array}$ & $\begin{array}{c}\text { Incl. } \\
\text { (degrees) }\end{array}$ & $\begin{array}{c}\text { Decl. } \\
\text { (degrees) }\end{array}$ & $\begin{array}{c}\mathrm{A} \\
\text { (degrees) }\end{array}$ & $\left(\begin{array}{c}\text { Intensity } \\
\left.\times 10^{-5} \frac{\mathrm{EMU}}{\mathrm{cc}}\right)\end{array}\right.$ & $\begin{array}{l}\text { MM } \\
(\%)\end{array}$ & \\
\hline \multicolumn{13}{|c|}{ SITE 169} \\
\hline $\begin{array}{l}7-1 \\
7-1 \\
7-1 \\
7-1 \\
7-1 \\
7-1 \\
7-1\end{array}$ & $\begin{array}{r}70^{*} \\
72^{*} \\
83^{*} \\
86^{*} \\
97^{*} \\
106^{*} \\
108^{*}\end{array}$ & $\begin{array}{r}-17.1 \\
-17.1 \\
-19.9 \\
-27.1 \\
16.9 \\
-14.1 \\
7.1\end{array}$ & $\begin{array}{r}52.6 \\
8.7 \\
208.8 \\
193.5 \\
225.2 \\
324.8 \\
295.5\end{array}$ & $\begin{array}{l}0.0 \\
0.1 \\
0.9 \\
4.2 \\
1.0 \\
0.4 \\
0.0\end{array}$ & $\begin{array}{l}96.8 \\
97.0 \\
74.1 \\
17.7 \\
24.6 \\
17.7 \\
50.1\end{array}$ & $\begin{array}{l}0.7 \\
0.9 \\
3 \\
7 \\
6 \\
7 \\
3\end{array}$ & $\begin{array}{l}-29.1 \\
-27.0 \\
-33.4 \\
-51.2 \\
-12.6 \\
-37.1 \\
-12.0\end{array}$ & $\begin{array}{r}56.8 \\
6.5 \\
208.8 \\
183.7 \\
234.0 \\
322.4 \\
280.2\end{array}$ & $\begin{array}{l}0.3 \\
0.0 \\
0.0 \\
1.0 \\
0.2 \\
0.5 \\
0.0\end{array}$ & $\begin{array}{l}71.6 \\
68.5 \\
48.0 \\
26.7 \\
21.1 \\
22.4 \\
25.7\end{array}$ & $\begin{array}{l}0.7 \\
0.7 \\
2 \\
3 \\
2 \\
3 \\
3\end{array}$ & $\begin{array}{l}\text { A } \\
\text { A } \\
\text { A } \\
\text { A } \\
\text { A } \\
\text { A } \\
\text { A }\end{array}$ \\
\hline \multicolumn{13}{|l|}{ SITE 170} \\
\hline $\begin{array}{l}6-4 \\
6-5 \\
6-5 \\
6-6 \\
6-6\end{array}$ & $\begin{array}{r}122^{*} \\
82^{*} \\
100^{*} \\
133^{*} \\
135^{*}\end{array}$ & $\begin{array}{r}-31.9 \\
-45.4 \\
10.0 \\
-9.3 \\
1.7\end{array}$ & $\begin{array}{r}231.5 \\
339.1 \\
227.8 \\
117.7 \\
97.9\end{array}$ & $\begin{array}{l}0.1 \\
5.9 \\
0.0 \\
0.2 \\
0.0\end{array}$ & $\begin{array}{r}950 . \\
24.8 \\
52.9 \\
72.1 \\
53.6\end{array}$ & $\begin{array}{c}1 \\
12 \\
0.9 \\
1 \\
0.8\end{array}$ & $\begin{array}{r}-31.5 \\
-48.3 \\
-2.8 \\
-20.6 \\
-15.7\end{array}$ & $\begin{array}{l}231.7 \\
307.7 \\
228.6 \\
118.1 \\
104.8\end{array}$ & $\begin{array}{l}0.6 \\
0.9 \\
0.1 \\
0.3 \\
0.5\end{array}$ & $\begin{array}{c}966 . \\
19.9 \\
38.7 \\
54.6 \\
39.2\end{array}$ & $\begin{array}{l}2 \\
2 \\
2 \\
0.8 \\
0.6\end{array}$ & $\begin{array}{l}\text { A } \\
\text { A } \\
\text { A } \\
\text { B } \\
\text { A }\end{array}$ \\
\hline $\begin{array}{l}7-3 \\
7-3 \\
7-3 \\
7-3 \\
7-3 \\
7-3 \\
7-3 \\
7-3 \\
7-3\end{array}$ & $\begin{array}{l}30^{*} \\
40^{*} \\
50^{*} \\
61^{*} \\
63^{*} \\
65^{*} \\
67^{*} \\
69^{*}\end{array}$ & $\begin{array}{r}1.9 \\
-6.1 \\
18.4 \\
6.1 \\
21.0 \\
-0.4 \\
10.3 \\
-2.8 \\
16.2\end{array}$ & $\begin{array}{r}86.3 \\
103.5 \\
183.5 \\
10.9 \\
179.9 \\
277.7 \\
232.5 \\
214.3 \\
148.3\end{array}$ & $\begin{array}{l}0.0 \\
0.2 \\
0.2 \\
0.0 \\
2.3 \\
0.2 \\
0.0 \\
0.0 \\
0.3\end{array}$ & $\begin{array}{r}152 . \\
51.6 \\
49.9 \\
49.4 \\
24.7 \\
90.2 \\
66.1 \\
73.5 \\
38.3\end{array}$ & $\begin{array}{l}2 \\
1 \\
2 \\
0.6 \\
6 \\
2 \\
2 \\
2 \\
3\end{array}$ & $\begin{array}{r}-9.3 \\
-24.1 \\
-1.4 \\
-14.0 \\
27.2 \\
-6.0 \\
12.6 \\
-14.3 \\
5.0\end{array}$ & $\begin{array}{r}85.1 \\
104.2 \\
190.2 \\
13.6 \\
123.9 \\
278.1 \\
228.4 \\
207.3 \\
136.3\end{array}$ & $\begin{array}{l}0.1 \\
0.4 \\
0.0 \\
0.1 \\
3.0 \\
0.0 \\
0.1 \\
0.1 \\
0.6\end{array}$ & $\begin{array}{r}124 . \\
43.1 \\
30.1 \\
34.5 \\
15.3 \\
66.3 \\
40.6 \\
46.2 \\
32.7\end{array}$ & $\begin{array}{l}1 \\
0.8 \\
3 \\
2 \\
5 \\
1 \\
2 \\
1 \\
2\end{array}$ & $\begin{array}{l}\text { A } \\
\text { C } \\
\text { C } \\
\text { B } \\
\text { B } \\
\text { B } \\
\text { B } \\
\text { B } \\
\text { B }\end{array}$ \\
\hline $\begin{array}{l}8-1 \\
8-1 \\
8-1 \\
8-1 \\
8-1 \\
8-1 \\
8-1 \\
8-1 \\
8-1 \\
8-1 \\
8-1\end{array}$ & $\begin{array}{r}41^{*} \\
59^{*} \\
80^{*} \\
100^{*} \\
106^{*} \\
108^{*} \\
114^{*} \\
116^{*} \\
122^{*} \\
124^{*} \\
126^{*}\end{array}$ & $\begin{array}{l}31.3 \\
31.5 \\
69.7 \\
80.8 \\
45.8 \\
47.0 \\
51.9 \\
67.1 \\
46.3 \\
33.2 \\
42.3\end{array}$ & $\begin{array}{l}331.1 \\
232.3 \\
242.3 \\
276.5 \\
263.0 \\
220.8 \\
108.9 \\
300.2 \\
299.4 \\
288.6 \\
284.7\end{array}$ & $\begin{array}{l}0.3 \\
0.5 \\
0.3 \\
0.1 \\
0.3 \\
1.1 \\
2.8 \\
0.1 \\
0.4 \\
0.0 \\
0.1\end{array}$ & $\begin{array}{c}66.8 \\
68.9 \\
45.6 \\
77.8 \\
61.7 \\
86.1 \\
32.9 \\
56.6 \\
109 . \\
104 . \\
87.8\end{array}$ & $\begin{array}{l}0.9 \\
1 \\
0.8 \\
1 \\
2 \\
3 \\
3 \\
3 \\
2 \\
2 \\
3\end{array}$ & $\begin{array}{r}12.5 \\
20.6 \\
47.3 \\
60.8 \\
53.7 \\
57.2 \\
17.3 \\
67.2 \\
41.1 \\
28.6 \\
-34.7\end{array}$ & $\begin{array}{r}323.1 \\
232.1 \\
210.9 \\
227.4 \\
262.9 \\
225.3 \\
77.2 \\
5.8 \\
318.8 \\
304.1 \\
286.4\end{array}$ & $\begin{array}{l}0.1 \\
0.5 \\
0.5 \\
0.3 \\
0.0 \\
0.0 \\
0.3 \\
0.4 \\
0.2 \\
0.1 \\
0.5\end{array}$ & $\begin{array}{l}47.2 \\
45.6 \\
21.6 \\
41.5 \\
36.6 \\
41.4 \\
25.2 \\
29.2 \\
71.1 \\
70.8 \\
54.1\end{array}$ & $\begin{array}{l}2 \\
2 \\
3 \\
0.4 \\
2 \\
1 \\
3 \\
3 \\
1 \\
1 \\
1\end{array}$ & $\begin{array}{l}\mathrm{A} \\
\mathrm{C} \\
\mathrm{C} \\
\mathrm{C} \\
\mathrm{C} \\
\mathrm{C} \\
\mathrm{C} \\
\mathrm{C} \\
\mathrm{C} \\
\mathrm{C} \\
\mathrm{C}\end{array}$ \\
\hline \multicolumn{13}{|c|}{ SITE 171} \\
\hline $\begin{array}{l}5-5 \\
5-5\end{array}$ & $\begin{array}{l}134 \\
136\end{array}$ & $\begin{array}{l}- \\
-\end{array}$ & $\begin{array}{l}- \\
-\end{array}$ & $\begin{array}{l}- \\
-\end{array}$ & $\begin{array}{l}- \\
-\end{array}$ & - & - & $\begin{array}{l}- \\
-\end{array}$ & $\begin{array}{l}- \\
-\end{array}$ & $\begin{array}{l}- \\
-\end{array}$ & $\overline{-}$ & $\begin{array}{l}\mathrm{A} \\
\mathrm{B}\end{array}$ \\
\hline $\begin{array}{l}22-1 \\
22-1 \\
22-1 \\
22-1 \\
22-1\end{array}$ & $\begin{array}{c}0^{*} \\
2^{*} \\
4^{*} \\
27 \\
29\end{array}$ & $\begin{array}{r}-26.5 \\
11.1 \\
8.7 \\
17.1 \\
-19.8\end{array}$ & $\begin{array}{r}195.4 \\
191.4 \\
3.1 \\
227.9 \\
327.4\end{array}$ & $\begin{array}{l}1.8 \\
1.6 \\
0.1 \\
0.0 \\
0.5\end{array}$ & $\begin{array}{l}6.1 \\
8.5 \\
9.4 \\
6.9 \\
8.8\end{array}$ & $\begin{array}{r}14 \\
10 \\
10 \\
5 \\
6\end{array}$ & $\begin{array}{r}-29.3 \\
1.0 \\
12.2 \\
12.3 \\
14.4\end{array}$ & $\begin{array}{l}194.6 \\
193.1 \\
187.5 \\
208.8 \\
202.4\end{array}$ & $\begin{array}{l}0.8 \\
2.3 \\
1.3 \\
0.6 \\
0.5\end{array}$ & $\begin{array}{r}4.2 \\
6.9 \\
7.5 \\
8.4 \\
11.1\end{array}$ & $\begin{array}{r}20 \\
12 \\
12 \\
6 \\
9\end{array}$ & $\begin{array}{l}E \\
E \\
E \\
E \\
E\end{array}$ \\
\hline
\end{tabular}




\begin{tabular}{|c|c|c|c|c|c|c|c|c|c|c|c|c|}
\hline 22-1 & 31 & 38.8 & 194.2 & 0.8 & 10.4 & 11 & 21.4 & 197.6 & 0.7 & 9.9 & 7 & $\mathrm{E}$ \\
\hline 22-1 & $81^{*}$ & 20.0 & 222.6 & 1.0 & 12.0 & 7 & 16.8 & 224.0 & 1.7 & 11.5 & 7 & $\mathrm{E}$ \\
\hline $22-1$ & 83* & 4.0 & 227.8 & 3.5 & 19.1 & 6 & 8.7 & 220.1 & 0.2 & 18.7 & 4 & E \\
\hline $22-1$ & $85^{*}$ & 12.8 & 213.1 & 0.8 & 18.7 & 6 & 13.3 & 214.5 & 0.5 & 18.4 & 3 & $\mathrm{E}$ \\
\hline 22-1 & $115^{*}$ & -42.2 & 189.3 & 1.0 & 12.4 & 12 & -20.9 & 164.3 & 0.1 & 9.4 & 8 & $\mathrm{E}$ \\
\hline $22-1$ & $117^{*}$ & 23.2 & 175.0 & 0.1 & 13.3 & 6 & 14.0 & 151.8 & 0.0 & 10.7 & 4 & E \\
\hline $22-1$ & $119 *$ & -12.9 & 180.1 & 0.1 & 9.8 & 10 & -14.3 & 156.7 & 0.4 & 9.2 & 6 & $\mathrm{E}$ \\
\hline $25-2$ & 5 & -25.6 & 359.4 & 14.8 & 1.5 & 21 & -26.0 & 4.6 & 5.0 & 1.1 & 36 & A \\
\hline $25-2$ & 7 & 15.2 & 21.8 & 12.0 & 1.0 & 20 & - & - & - & - & - & A \\
\hline $26-3$ & $43^{*}$ & -6.2 & 209.3 & 0.1 & 404. & 1 & -12.3 & 211.2 & 0.1 & 343. & 2 & A \\
\hline $26-3$ & $45^{*}$ & -3.4 & 210.7 & 0.0 & 206. & 1 & -8.3 & 215.2 & 0.0 & 191. & 0.7 & A \\
\hline $26-3$ & 119 & -5.5 & 296.8 & 1.1 & 1.5 & 15 & -19.4 & 306.4 & 10.6 & 0.9 & 41 & B \\
\hline
\end{tabular}

NOTE: Asterisk denotes samples run on Stanford spinner (see text). Dashes indicate that sample was too weak to measure.

asee text for definitions of letters A through $\mathrm{E}$

b Broken sample.

${ }^{c} 14 c c$ cylindrical samples too large for demagnetization apparatus. 
R. D. JARRARD

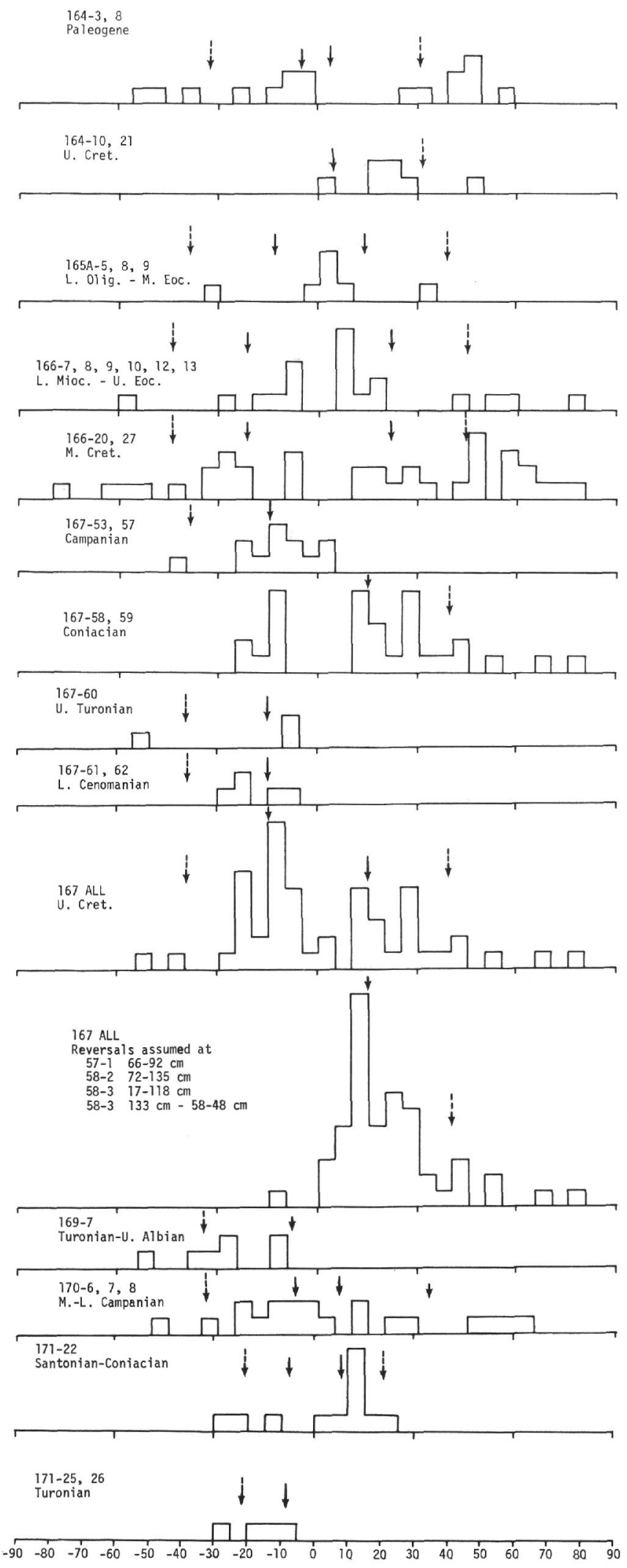

Figure 2. Histograms of inclinations of the magnetization vectors in sediments after 50 oersted A. F. demagnetization. Solid arrows indicate expected average inclinations for each site if $15^{\circ}$ of northward motion of the sea floor has occurred since deposition; dashed arrows correspond to $30^{\circ}$ of northward motion. The age of the sediments within each histogram is indicated beneath the site and core numbers. 


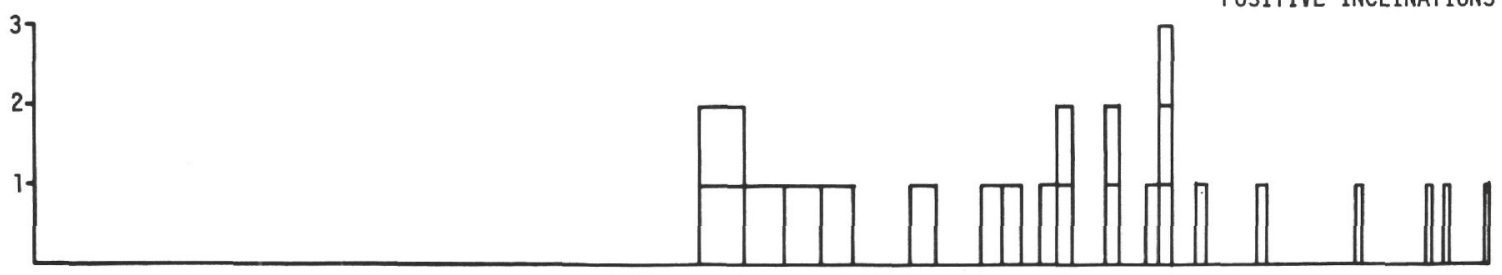

NEGATIVE INCLINATIONS

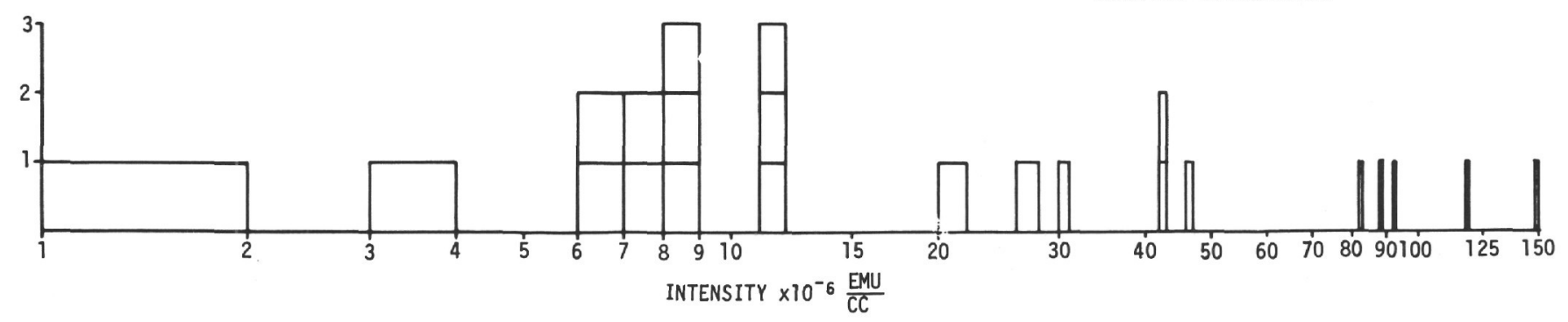

Figure 3. Histograms of intensities of samples separated according to the sign of the sample inclination. Horizontal scale is logarithmic. Each rectangle represents one sample.

\section{ACKNOWLEDGMENTS}

I wish to thank John Sclater, who pointed out to me the potential of inclinations of deep-sea sediments and encouraged me to pursue this work. Paul Liebertz made most of the paleomagnetic measurements. Allan Cox generously offered me the use of his spinner magnetometer and a computer program for working up the data, and Charles Denham introduced me to that spinner.

\section{REFERENCES}

Francheteau, J., Harrison, C. G. A., Sclater, J. G., and Richards, M., 1970. Magnetization of Pacific seamounts: a preliminary polar curve for the north eastern Pacific: J. Geophys. Res., V. 75, p. 2035-2061.

Grommé, S. and Vine, F. J. Paleomagnetism of Midway Atoll lavas and northward movement of the Pacific plate: Earth Planet. Sci. Lett. (in press).
Helsley, C. E. and Steiner, M. B., 1969. Evidence for long intervals of normal polarity during the Cretaceous period: Earth Planet. Sci. Lett., v. 5, p. 325-332.

Larson, R. L. and Chase, C. G., 1972. Late Mesozoic evolution of the western Pacific: Geol. Soc. Am. Bull., v. 83, p. 3627-3644.

McKenzie, D. and Sclater, J. G., 1971. The evolution of the Indian Ocean since the Late Cretaceous: Roy. Astron. Soc. Geophys. J., v. 25, p. 437-528.

Sclater, J. G. and Cox, A., 1970. Paleolatitudes from JOIDES deep sea sediment cores: Nature, v. 226, p. 934-935.

Sclater, J. G. and Jarrard, R. D., 1971. Preliminary paleomagnetic results, Leg 7: Initial Reports of the Deep Sea Drilling Project, Volume VII. Washington (U. S. Government Printing Office) p. 1227-1234.

Vine, F. J., 1968. Paleomagnetic evidence for the northward movement of the North Pacific Basin during the past 100 m.y. (abstract): Am. Geophys. Union Trans., v. 49 , p. 156. 


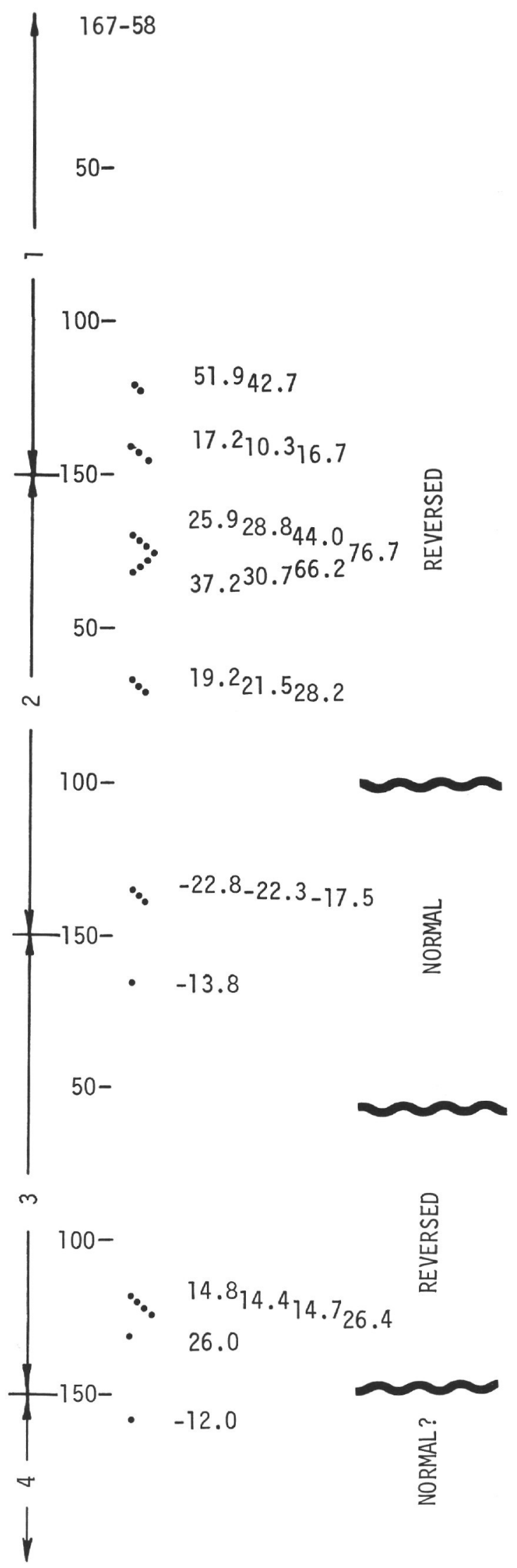

Figure 4. Identification of reversals in Coniacian Core 58 at Site 167. 